\title{
Estabilidad
}

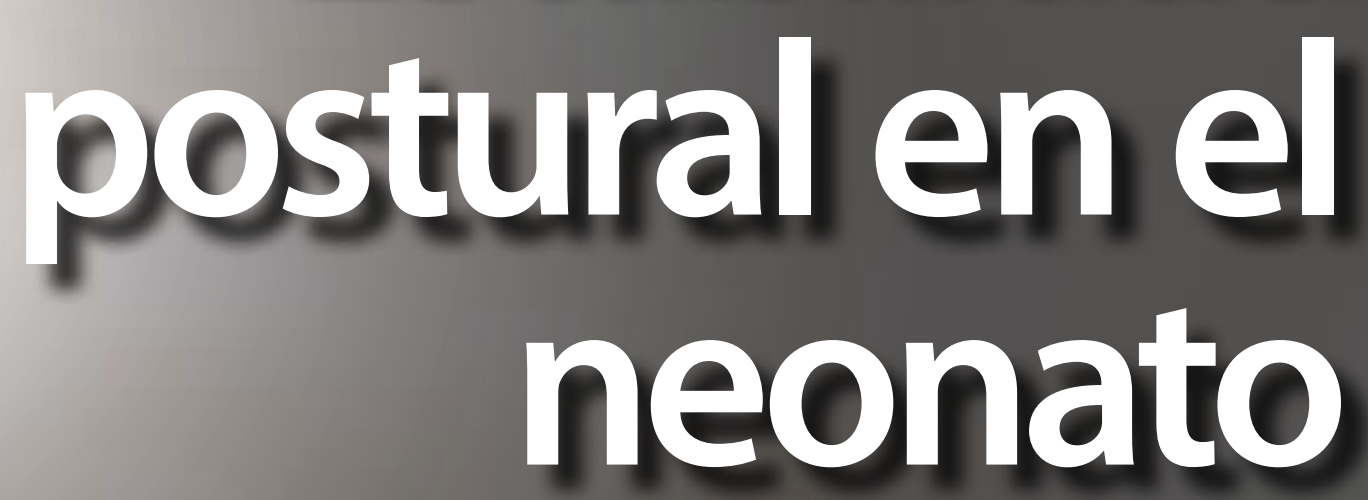

\section{Una revisión de la literatura}

Postural stability in the neonate. Review of literatura

(c) $\frac{1}{\mathrm{BY}}$ () (2)

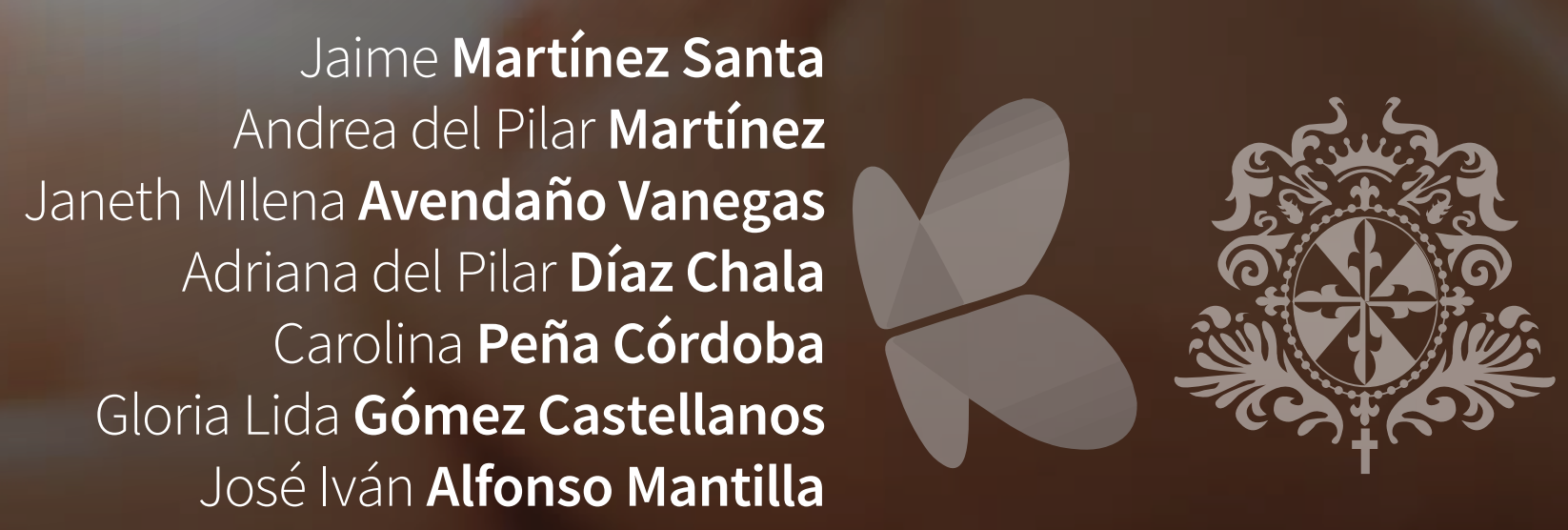

Movimiento Cientifico 
Movimiento Cientifico

\section{ISSN-l: 2011-7191 | e-ISSN: 2463-2236 Publicación Semestral}

ID:

2011-7191.mct.13207

Title: $\quad$ Postural stability in the neonate. Review of literatura

Título: Estabilidad postural en el neonato: Una revisión de la literatura

Alt Title / Título alternativo:

[en]: Postural stability in the neonate. Review of literatura

[es]: $\quad$ Estabilidad postural en el neonato: Una revisión de la literatura

Author (s) / Autor (es):

Martínez Santa, Martínez, Avendaño Vanegas, Díaz Chala, Peña Córdoba, 'Gómez Castellanos, \& Alfonso Mantilla

Keywords / Palabras Clave:

[en]: Newborn; Postural Balance; Child Development

[es]: $\quad$ Recién Nacido; Balance postural; Desarrollo infantil

Submited: $\quad$ YYYY-01-18

Acepted: $\quad$ YYYY-09-12

\section{Resumen}

Introducción La estabilidad postural se define como aquella habilidad para regular el centro de masa en relación a la base de sustentación. La estabilidad postural estática hace alusión al balance postural, entendido este como aquella armonía entre las fuerzas de inercia que actúan sobre el cuerpo (externas) y las que actúan desde los segmentos corporales (internas)

Objetivo Realizar una revisión de literatura sobre

los procesos que llevan a cabo los sistemas de

retroalimentación sensorial para alcanzar la estabilidad postural en el neonato. Metodología Se efectuó una búsqueda de evidencia en bases de datos como Science Direct, Elsevier, Springer, Annual Reviews, entre los años 1990 y 2018 con

los siguientes términos DeCS: Recién Nacido, Balance postural, Desarrollo infantil. Resultados Se obtuvieron 53 artículos donde se determinó que la estabilidad postural es un concepto que procede de 2 orígenes, el primero relacionado con los sistemas sensoriales (somatosensorial, vestibular y visual) los cuales conllevan a variaciones en las oscilaciones del centro de gravedad. El segundo está relacionado con las adaptaciones biomecánicas presentes. El desarrollo postural

en el neonato comienza con un repertorio de ajustes específicos que sugieren un origen innato. Conclusión El control postural en el neonato se da en una representación cefalocaudal teniendo un importante papel la cabeza, el cuello y el tronco. El sistema vestibular está conectado con

los sistemas propioceptivo, visual y motor en la adquisición del control postural, la conexión entre el sistema visual y el sistema vestibular es alcanzada entre el segundo y tercer mes de vida.

\section{Abstract}

Introduction Postural stability is defined as the ability to regulate the center of mass in relation to the base of support. Static postural stability refers to the postural balance, understood as the harmony between the forces of inertia that act on the body (external) and those that act from the body segments (internal) Objective To conduct a literature review on the processes that lead out the sensory feedback systems to achieve postural stability in the neonate.

Methodology A search of evidence was

made in databases such as Science Direct, Elsevier, Springer, Annual Reviews, between the years 1990 and 2018 with the following DeCS terms: Newborn, Postural Balance, and Child Development. Results

We obtained 53 articles where it was possible to determine that postural stability is a concept that comes from 2 origins, the first one related to sensory systems: (somatosensory, vestibular and visual) which lead to variations in the oscillations of the center of gravity. The second is related to the biomechanical adaptations present. The postural development in the neonate begins with a repertoire of specific adjustments that suggest that the basic level of control has an innate origin. Conclusion The postural control in the neonate occurs in a cephalocaudal representation having an important role, the head, the neck and the trunk. The vestibular system is connected to the proprioceptive, visual and motor systems in the acquisition of postural control, the connection between the visual system and the vestibular system is reached between the second and third month of life.

\section{Citar como:}

Martínez Santa, J., Martínez, A. d., Avendaño Vanegas, J. M., Díaz Chala, A. d., Peña Córdoba, C.,

'Gómez Castellanos, G. L., \& Alfonso Mantilla, J. I. (2019). Estabilidad postural en el neonato: Una revisión de la literatura. Revista Iberoamericana de Psicología issn-l:2011-7191, 13 (2), 19-26.

Obtenido de: https://revmovimientocientifico.ibero.edu.co/article/view/1540

\author{
Jaime Martínez Santa, Ft. \\ Source | Filiacion: \\ Colsanitas Clínica Colombia Unidad de \\ Recién Nacidos \\ BIO: \\ Fisioterapeuta \\ City | Ciudad: \\ Bogotá [co] \\ e-mail: \\ jmartinezsanta@yahoo.com \\ Andrea del Pilar Martínez, Ft. \\ Source | Filiacion: \\ Colsanitas Clínica Colombia Unidad de \\ Recién Nacidos \\ BIO: \\ Fisioterapeuta \\ City | Ciudad: \\ Bogotá [co]
}

Janeth Mllena Avendaño Vanegas, Ft. Source | Filiacion:

Colsanitas Clínica Colombia Unidad de Recién Nacidos

BIO:

Fisioterapeuta

City | Ciudad:

Bogotá [co]

Adriana del Pilar Díaz C
Source | Filiacion:
Colsanitas Clínica Colom
Recién Nacidos
BIO:
Terapeuta Respiratoria
City | Ciudad:
Bogotá [co]

Adriana del Pilar Díaz Chala, Tr

Source Filiacion:

BIO:

City | Ciudad:

Carolina Peña Córdoba, Ft.
Source | Filiacion:
Universidad del Rosario
BIO:
Docente, Universidad del Rosario, Fisiote-
rapeuta
City | Ciudad:
Bogotá [co]
Gloria Lida Gómez Castellanos, Ft.
Source | Filiacion:
Universidad del Rosario
BIO:
Docente, Universidad del Rosario, Fisiote-
rapeuta
City | Ciudad:
Bogotá [co]
e-mail:
gloria.gomez@ibero.edu.co

José Iván Alfonso Mantilla, Ft.

\section{Source | Filiacion:}

Universidad del Rosario

BIO:

Fisioterapeuta

City | Ciudad:

Bogotá [co]

e-mail:

josealfonso25@hotmail.com 


\title{
Estabilidad postural en el neonato Una revisión de la literatura
}

Postural stability in the neonate. Review of literatura

\author{
Jaime Martínez Santa \\ Andrea del Pilar Martínez \\ Janeth Mllena Avendaño Vanegas \\ Adriana del Pilar Díaz Chala \\ Carolina Peña Córdoba \\ Gloria Lida Gómez Castellanos \\ José Iván Alfonso Mantilla
}

\section{Introducción}

En la actualidad, la fisioterapia se ha convertido en un área de estudio del desarrollo motor del infante por lo cual estudia elementos tales como la estabilidad postural, la cual es entendida como aquella habilidad para regular el centro de masa en relación a la base de sustentación (ShumwayCook \& Wollacott, 1995). La estabilidad postural estática hace alusión al balance postural, entendido como aquella armonía entre las fuerzas de inercia que actúan sobre el cuerpo (externas) y las que actúan desde los segmentos corporales (internas) (Winter, 1995)

El nivel básico de estabilidad tiene que ver con la generación de ajustes en direcciones específicas, lo que significa que la musculatura dorsal se activa principalmente cuando el cuerpo se mueve hacia delante y viceversa. El segundo nivel, está involucrado en la adaptación de estos ajustes. El desarrollo postural comienza con un repertorio de ajustes específicos de dirección que sugieren que el nivel básico de control tiene un origen innato. (Hadders-Algra, 2005)

Para lograr una adecuada estabilidad postural se habla de procesos que llevan a cabo los sistemas sensoriales, motores y cognitivos; dentro del sistema sensorial se destacan los procesos mediados a través de los sistemas somatosensorial, visual y vestibular. (Horak, 2006). A nivel somatosensorial encontramos la propiocepción entendida como una "modalidad sensorial que abarca las sensaciones del movimiento articular y la posición articular, contribuyendo a la programación motora y respuestas musculares para la estabilidad postural" (Peterka, 2002) (Caniuqueo, Fernandes, Quiroz, \& René, 2014 ). El sistema visual hace parte de los sistemas encargados de lograr una adecuada estabilidad postural, ya que su función es detectar los movimientos y el ambiente que rodea al sujeto. (Caniuqueo, Fernandes, Quiroz, \& René, 2014 ) (Peterka, 2002). El sistema vestibular, es el último sistema que provee información de cómo se mueve el cuerpo en el espacio (Peterka, 2002) (Lipsitz, 2002) (Caniuqueo, Fernandes, Quiroz, \& René, 2014) 


\section{Estabilidad postural en el neonato}

Una revisión de la literatura

El presente artículo tiene como fin hacer un énfasis al trabajo conjunto de los procesos que llevan a cabo los sistemas de orden sensorial como parte de la integración de información para lograr la correcta estabilidad postural en el neonato que es vital importancia para el correcto desarrollo de habilidades motoras para la consecución de actividades funcionales en el infante. El objetivo de este trabajo fue realizar una revisión de literatura sobre los procesos que llevan a cabo los sistemas de retroalimentación sensorial para alcanzar la estabilidad postural en el neonato.

\section{Materiales y métodos}

Se efectúo una revisión de la literatura con la búsqueda de artículos científicos en bases de datos como: PubMed, Science Direct, Elsevier, Annual Reviews y Springer teniendo en cuenta los siguientes términos DeCs Newborn, Postural Balance, and Child Development. Adicionalmente, se tuvieron en cuenta los siguientes criterios de inclusión y exclusión

Figura 1 (Flujograma de extracción de la evidencia)

Búsqueda en bases de datos:

PubMed, Science Direct, Elsevier, Annual Review y Springer

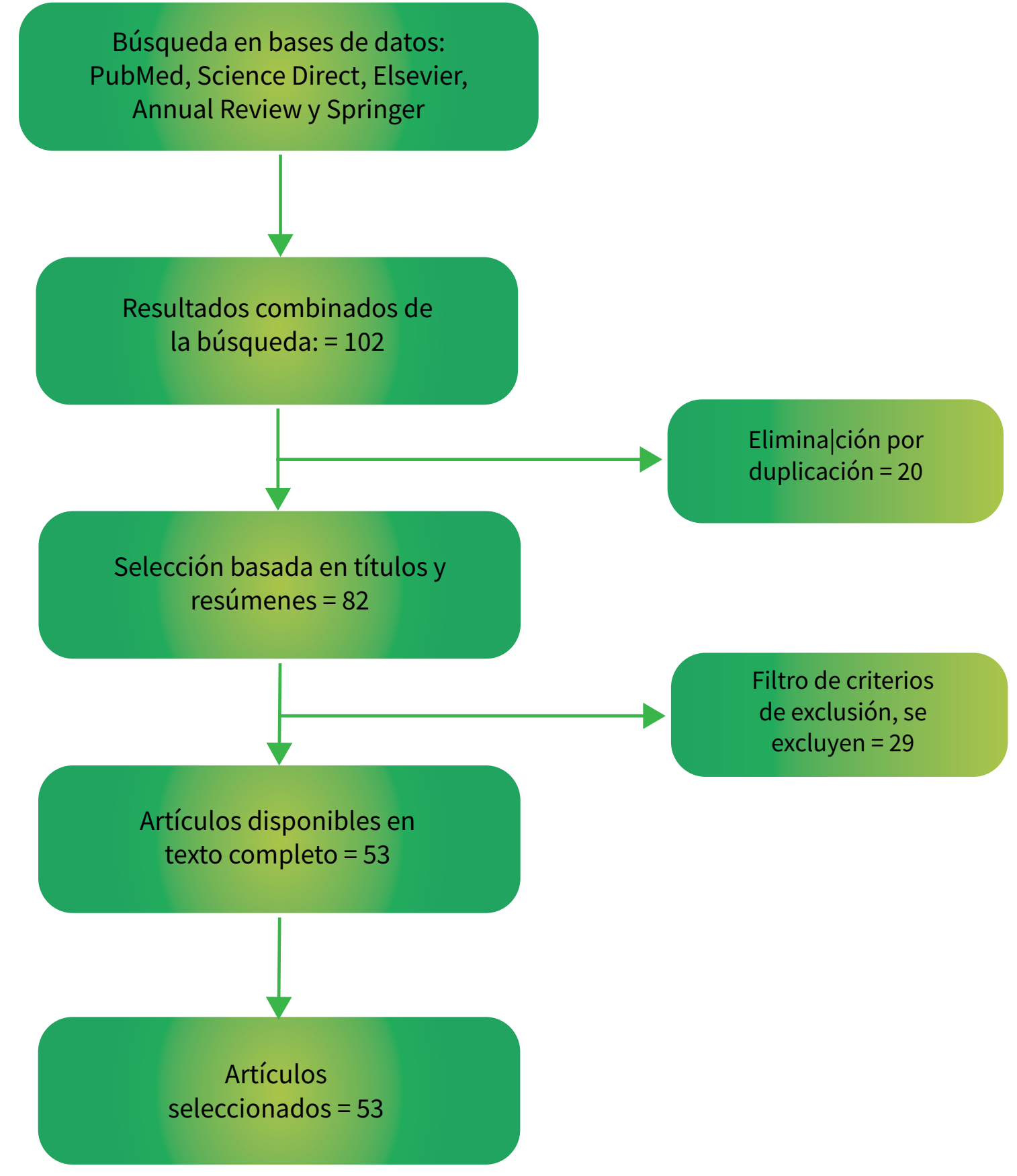

\section{Criterios de inclusión}

$\rightarrow$ Estudios publicados entre 1990 y 2018.

$\rightarrow$ Estudios que contemplaran los términos DeCs.

$\rightarrow$ Idioma español, inglés y portugués.

\section{Criterios de exclusión}

$\rightarrow$ Estudios que su año de publicación fuera inferior a 1990

$\rightarrow$ Estudios que no estuvieran disponibles para la lectura por parte de los autores.

\section{Resultados}

Una vez aplicados los criterios de inclusión y exclusión se obtuvo un total de 53 artículos de los cuales 13 fueron analíticos y de corte experimental y los 40 restantes correspondieron a revisiones de la literatura y estudios descriptivos. De acuerdo con esta revisión, se encontró que el $42 \%$ de los artículos, eran procedentes de Estados Unidos, 10\% de Canadá, 10\% de Francia, 8\% de España y el 30\% restante corresponde a otros países como para Perú, Países Bajos, Australia, Reino Unido e India, esto se resumen en la figura 1 (Extracción de la evidencia) 
Dentro de la evidencia se pudo identificar la importancia de la estabilidad postural en el neonato y sus adaptaciones neurofisiológicas y sensoriales.

\section{Estabilidad postural en el neonato}

Existen receptores especializados en la captación de estímulos externos en relación a la posición del cuerpo en el espacio: son los mecanorreceptores (componentes base delsistemasomatosensorial) quienes cumplen la función de codificarinformación sobrela posición y orientación corporal a través de la aceleración gravitacional que emite el sistema vestibular en conjunto con el sistema visual lo que establece una orientación clara del ambiente y los segmentos corporales (Horak, 2006) (Prieto, Myklebust, Hoffmann, Lovett, \& Myklebust, 1996)

Dicha información sensorial converge en el sistema nervioso central con la finalidad de que los centros superiores emitan reacciones para compensar el resultado de alteraciones en la actitud postural. Mediante la experiencia, los neonatos aprenden a mantener el control postural mientras interactúan dentro de un entorno, esto requiere una compleja interacción de sistemas en desarrollo que incluyen los sistema sensorial, motor y cognitivo. (Goldfield, 1995) (Oliveira, Vieira, Macedo, Simpson, \& Nadal, 2009) (Moccellin \& Driusso, 2012) (Dusing, Thacker, \& Galloway, 2016)

El control postural difiere entre los recién nacidos a término y pretérmino durante las 3 primeras semanas de vida, no es claro si esas diferencias continúan y si tienen efectos en el desarrollo de los comportamientos de los niños. Los recién nacidos pretérmino tienen un mayor riesgo de desarrollar discapacidades que incluyen parálisis cerebral, desarrollo de desórdenes de coordinación y dificultades para el aprendizaje, comparados con los recién nacido a término. (Stacey, 2014).

\section{Adaptaciones del sistema}

\section{somatosensorial en el recién nacido}

El propósito de las adaptaciones del sistema somatosensorial no es solamente especificar las posiciones respectivas de los segmentos corporal sino también especificar la orientación postural del cuerpo con respecto al mundo externo (Mergner, Hlavacka, \& Schweigart, 1993)

Esta orientación esta monitorizada por los propioceptores localizados en el cuello (otolitos), en el tronco (propioceptores alrededor del hígado) (Mittelstaedt \& Glasauer, 1993) (Mittelstaedt, 1995) en las articulaciones (receptores de carga con información acerca del eje vertical de gravedad) y en el pie (receptores plantares). La interpretación de la información propioceptiva obtenida depende de cual segmento es usado como referencia con respecto al mundo externo.

El control postural muestra un desarrollo cefalocaudal con respuestas posturales que aparecen primero en cuello y tronco, finalmente en los miembros inferiores entre los 3 y los 14 meses de edad con un amplio patrón de respuestas que antes de la experiencia con la estabilización del centro de masa cuya proyección debe permanecer dentro de la zona de soporte para mantener el equilibrio que en condiciones estáticas (Hadders-Algra, Brogren, \& Forssberg, 1996) .Durante la vida intrauterina, el vector de gravedad está presente, sin embargo, el feto vive en un ambiente acuático, donde los receptores de carga no están monitoreando peso; la adaptación al peso es por lo tanto, la tarea principal del sistema sensorio motor después del nacimiento. Por esta razón la estrategia más efectiva en el neonato es reducir la variabilidad postural o la magnitud del desplazamiento del centro de masa en direcciones medial lateral y caudal cefálico (Manssion, 1998) (Halek, y otros, 2015) (Dusing, Thacker, \& Galloway, 2016).

La activación postural ocurre antes de los desajustes relacionados con el movimiento de los miembros superiores y puede ser calificado como ajustes posturales anticipatorios los cuales utilizan los recién nacidos (Van Der Fits \& Hadders-Algra, 1998). La precisión con la que un niño o un adulto puede equilibrarse durante la postura tranquila y el movimiento voluntario depende de la detección e integración eficientes de la información de los sistemas somato sensorial, visual y vestibular, así como la consiguiente activación de respuestas musculares organizadas de manera adecuada. (Sveistrup \&Woollacott, 1996).

\section{Adaptaciones del sistema visual en el recién nacido}

La cabeza con sus propios receptores visuales y vestibulares es la primera parte del cuerpo en desarrollar una orientación postural. Al final del primer año de vida el control cefálico es el más avanzado de todos los segmentos del cuerpo. Por esta razón la postura de la cabeza en el espacio contribuye de manera importante a la postura de los segmentos inferiores del cuerpo con respecto al mundo externo y el modo descendente de la organización postural; esto también explica el predominio temprano de la visión en la postura y el hecho de que los niños permanecen durante mucho tiempo dependientes de la estimulación visual. (Manssion, 1998).

El mecanismo primario de adaptación al cambio de los niveles de luz en el neonato es la adaptación pupilar y el sistema dual de receptores diseñados para la luz del día (conos) y la visión nocturna o con baja intensidad de la luz (bastones). Durante la vida fetal y el periodo neonatal la retina crece a la par con el crecimiento ocular migrando los bastones a la periferia y los conos al área central de la retina, este proceso no está relacionado con la presencia de estímulos visuales (Hadders-Algra, 2002) (Kolb, 2003) (Fielder,2004) (Reeves, 2004) (Graven, 2004). cuyos receptores transmiten a la corteza información relacionada con forma, movimiento, visión binocular y color sin existir una separación funcional (Palmer, 1999).

El propósito de esta actividad es desarrollar una topografía básica y crear las conexiones que se necesitan para preparar a los sistemas a un desarrollo neurosensorial más específico, este proceso es esencial para el desarrollo neurosensorial el cual incluye al sistema somatosensorial, el movimiento, la visión y la audición (White-Traut, Nelson, Burns, \& Cunningham, 1994) (Wharrad \& Davis, 1997 ) (Penn \& Riquelme PA, 1998) (Penn \& Shatz, 1999) (Penn, 2001) (Penn \& Shatz, 2002) (Sranley, 2004). El sistema visual del neonato se va a desarrollar como dependiendo del ambiente y sus entradas sensoriales que ayudan a refinar las distribuciones topográficas (mapas) y las características de visión de cada recién nacido incluyendo la relación entre la visión de color y la visión espacial. (Brown, 1990) (Allen, Banks, Norcia, \& Shannon, 1993) (Morrone, Burr, 


\section{Estabilidad postural en el neonato}

\section{Una revisión de la literatura}

\& Fiorentini, 1993) (Adams, Maurer, \& \& Cashin, 1990). (Dobkins, Lia,, \& Teller, 1997) (Wilson, 1993 ) (Shannon, Skoczenski, \& Banks, 1996)

En los bebes nacidos a término, el control postural adaptativo permite a los neonatos ajustar su estrategia de control postural a las cambiantes demandas de tareas desde supino. Estos bebés sanos redujeron su variabilidad postural o la magnitud de su desplazamiento del centro de presión cuando se presenta con un estímulo visual. Esta respuesta adaptativa demuestra la flexibilidad en el sistema de control postural lo cual permite al niño aprender de su entorno (Dusing, Thacker, \& Galloway, 2016). Los recién nacidos prematuros no alteraron la variabilidad postural en respuesta a un estímulo visual antes de los 4 meses de edad en la dirección cefálica caudal. Sin embargo, son capaces de adaptar su variabilidad postural en la dirección lateral medial a los 2.5 meses de edad cuando se les presentó un estímulo visual (Babik, Galloway, \& Lobo, 2017).

Adquirir de manera temprana los movimientos de alcance está acompañado de patrones posturales complejos de respuesta que se asemejan a la postura adulta en las características espaciales (orden dorso-ventral), características temporales (secuencia cefalocaudal), y dependencia de la posición. Esto sugiere que los mecanismos epigenéticos desempeñan un papel importante en la ontogenia de los ajustes posturales que acompañan a los movimientos de alcance (Van Der Fits \& Hadders-Algra, 1998). Los primeros patrones posturales se asemejaban a los de los adultos en las características espaciales (ordenamiento dorsoventral), características temporales (reclutamiento descendente) y dependencia de posición. La anticipación postural durante el alcance no estuvo constantemente presente a ninguna edad (Carvalho, 2007).

Aunque en el nacimiento los bebés son capaces de iniciar los movimientos de cabeza y movimientos oculares, a los 2 meses usan movimientos oculares exclusivamente para ver objetivos periféricos estacionarios, mientras que a los 3 meses los bebés coordinan movimientos de la cabeza y los ojos para capturar objetivos visuales acercándose al patrón adulto de coordinación cabeza-ojo (Haith, Wentworth, \& Canfield, 1993 ). Los movimientos reflejos y no reflejos de la cabeza ocurren desde el nacimiento y el nistagmo vestibular en recién nacidos y niños pequeños es cualitativamente comparable al reflejo adulto (Canfield, y otros, 1997).

La coordinación cabeza-ojo involucra el movimiento visual, vestibular, de la cabeza y sistemas de movimiento ocular. Los recién nacidos pueden producir movimientos de cabeza y ojos, y muestran reflejos vestibulooculares claros. Sin embargo, la coordinación entre el sistema vestibular y los sistemas de movimiento de cabeza y ojo de forma madura no aparece hasta el segundo o tercer mes de vida (Babik, Galloway, \& Lobo, 2017).

\section{Adaptaciones del sistema vestibular en el recién nacido}

Los estudios neurofisiológicos indican que el sistema vestibular está íntimamente conectado con los sistemas propioceptivo, visual y motor en la adquisición de los reflejos del desarrollo y el control postural (Assaiante, Mallau, Viel, Jover, \& Schmitz, 2005) (de Klejn 1924, Magnus 1924) además de ser un indicador de maduración del sistema nervioso central.

El control postural es importante para el desarrollo de todos los movimientos, el balance y la locomoción, dependiendo en gran medida del componente vestibulospinal en los primeros años de vida (de Graaf, y otros, 2004).

Está claro que la comunicación entre los sistemas visual, vestibular, de movimiento de la cabeza y del movimiento ocular se vuelve más efectivo entre el segundo y el tercer mes de vida. A pesar de que la coordinación cabeza-ojo sin duda experimenta un mayor desarrollo después del tercer mes de vida, el circuito neuronal básico parece ser funcional hasta esa edad. (Babik, Galloway, \& Lobo, 2017).

La integración de estos dos sistemas ayuda a construir junto con el sistema somatosensorial un repertorio de estrategias posturales que le permiten al niño obtener un control del balance y mejor eficiencia en sus tareas motoras (Assaiante, Mallau, Viel, Jover, \& Schmitz, 2005).

\section{Discusión}

Los neonatos aprenden la estrategia de control postural disponible a través de la experiencia durante los periodos tempranos de más alta complejidad y retienen la capacidad de seleccionar múltiples estrategias, pero usan un pequeño número en una base regular (Massion, Alexandrov, \& Frolov, 2004). El uso de estrategias de control postural, permite al neonato experimentar el movimiento e interactuar con el ambiente desde múltiples puntos de vista usando acciones que proporcionan entradas perceptuales variadas, gracias a la adaptación de los sistemas en desarrollo que incluyen los sistema sensorial, motor y cognitivo. La complejidad postural temprana en el desarrollo proporciona al neonato una variedad de experiencias desde interacciones entre su cuerpo y el ambiente lo cual promueve el aprendizaje incorporado (Dusing, Izzo, Thacker, \& Galloway, 2014).

Como se pudo apreciar a lo largo de los resultados la estabilidad postural es el desarrollo de un conjunto de factores relacionados con el movimiento corporal humano. Se debe seguir haciendo investigación en el área con el fin de identificar factores relacionados con el desarrollo a nivel motor y sensorial del recién nacido que permitan realizar detecciones de alteraciones a nivel funcional que repercutan en el desarrollo de las actividades de la vida diaria de los recién nacidos a futuro y puedan implicar un factor negativo en la adquisición de habilidades relacionadas con el crecimiento y desarrollo motor. El fisioterapeuta debe ser un profesional que desarrolle investigaciones generando indicadores de seguimiento desde edades tempranas con el fin de detectar alteraciones en el movimiento corporal humano.

\section{Conclusiones}

El control postural en el neonato se da en una representación cefalocaudal teniendo inicialmente un importante papel, la cabeza, el cuello y el tronco. En la cabeza están los órganos visuales, el laberinto y el cuello con sus propioceptores que son el origen de los reflejos cervicales y laberinticos. En el tronco están los propioceptores alrededor del hígado originando los reflejos posturales a nivel lumbar

Las adaptaciones a nivel visual permiten crear las conexiones que se necesitan para preparar a los sistemas a un desarrollo neurosensorial más específico, incluyendo al sistema somatosensorial, la visión y la audición 
El sistema vestibular está íntimamente conectado con los sistemas propioceptivos, visual y motor en la adquisición de los reflejos del desarrollo y el control postural, la conexión entre el sistema visual y el sistema vestibular no se da en el neonato y es alcanzada entre el segundo y tercer mes de vida

\section{Referencias}

Adams, R., Maurer, D., \& \& Cashin, H. (1990). The influence of stimulus size on newborns' discrimination of achromatic stimuli. Vision research, 30(12), 2023-2030

Allen, D., Banks, M., Norcia, A., \& Shannon, L. (1993). Does chromatic sensivity develop more slowly than luminance sensivity? Vision Research-oxford, 33 2553-2553.

Assaiante, C., Mallau, S., Viel, S., Jover, M., \& Schmitz, C. (2005). Development of postural control in healthy children: a function approach. Neural plasticity, 12, 109-118.

Babik, I., Galloway, J., \& Lobo, M. (2017). Infants Born Preterm Demonstrate Impaired Exploration of Their Bodies and Surfaces Throughout the First 2 Years of Life. Physical Therapy, 97(9), 915-925.

Canfield, R., Smith, E., Brezsnyak, M., Snow, K., A. R., Haith, M., \& Adler, S. (1997). Information processing through the first year of life: A longitudinal study using the visual expectation paradigm. Monographs of the society for research in child development, i- 160.

Caniuqueo, A., Fernandes, J., Quiroz, G., \& René, R. (2014 ). Cinética de marcha, balance postural e índice de masa corporal durante el primer, segundo y tercer trimestre de Embarazo. Revista peruana de Ginecología y Obstetricia, 109-115.

de Graaf, M., Samsom, J., Pettersen, E., Schaaf, V., van Schie, P., \& de Groot, L. (2004). Vestibulospinal component of postural control (vestibular function) in very preterm infants (25 to 27 weeks) at 3, 6, and 12 months corrected age. Journal of child neurology, 19(8), 614-618.

Dobkins, K., L. B., \& Teller, D. (1997). Infant Color Vision: Temporal Contrast Sensitivity Functions for Chromatic (Red/Green). Vision Res, 37(19), 26992716.

Dusing, S., Izzo, T., Thacker, L., \& Galloway, J. (2014). Postural complexity differs between infant born full term and preterm during the development of early behaviors. Early human development, 90 (3), 149-156.

Dusing, S., Thacker, L., \& Galloway, J. (2016). Infant born preterm have delayed development of adaptive postural control in the first 5 months of life. Infant Behavior and Development, 49-58.

Goldfield, E. (1995). Emergent forms: Origins and early development of human action and perception. Oxford University Press on Demand.

Graven, S. (2004). Early neurosensory visual development of the fetus and newborn. . Clinics in perinatology, 31(2), 199-216.

Hadders-Algra, M. (2002). Variability in infant motor behavior: a hallmark of the healthy nervous system. Infant Behav Dev, 25(4), 433-51.

Hadders-Algra, M. (2005). Development of postural control during the first 18 months of life. Neural plasticity, 12(2-3), 93-108.

Hadders-Algra, M., Brogren, E., \& Forssberg, H. (1996). Ontogeny of postural adjustments during sitting in infancy: variation, selection and modulation. The Journal of Physiology,, 496(1), 273-288.

Haith, M., Wentworth, N., \& Canfield, R. (1993 ). The formation of expectations in early infancy. Advances in infancy research

Halek, J., Muckova, A., Svoboda, Z., Janura, M., Marikova, J., Horakova, K., \& Nemcova, N. (2015). Kinematic analysis of preterm newborns' spontaneous movements for postural activity assessment. Biomed Pap Med Fac Univ Palacky Olomouc Czech Repub, 159(4), 657-660.

Horak, F. (2006). Postural orientation and equilibrium: What do you need to know about neural control of balance to prevent falls? Oxford University Press on Behalf of the British Geriatrics Society, 7-11.
Kolb, H. (2003). How the retina works-much of the construction of an image takes place in the retina itself through the use of specialized neural circuits. Am Sc, 28-35.

Lipsitz, L. (2002). Dynamics of stability: the physiologic basis of functional health and frailty. J Gerontol A biol Sci Med Sci, 115-125

Manssion, J. (1998). Postural control systems in developmental perspective. Neuroscience \& Biobehavioral Reviews, 22(4), 465-472

Massion, J., Alexandrov, A., \& Frolov, A. (2004). Why and how are posture and movement coordinated. In Progress in brain research, 143, 13-27.

Mergner, T., Hlavacka, F., \& Schweigart, G. (1993). Interaction of vestibular and proprioceptive inputs. Journal of Vestibular Research, 3, 41-57.

Mittelstaedt, H. (1995). The formation of the visual and the postural vertical. In Multisensory Control of Posture. New York/London: T. Mergner and F. Halvacka.

Mittelstaedt, H., \& Glasauer, S. (1993). Crucial effects of weightlessness on human orientation. Journal of Vestibular Research, 307-314

Moccellin, A., \& Driusso, P. (2012). Adjustments in static and dynamic postura control during pregnancy and their relationship with quality of life: A descriptive study. Fisioterapia, 34(5), 196-202.

Morrone, M., Burr, D., \& Fiorentini, A. (1993). Development of infant contrast sensitivity to chromatic stimuli. Vision Research, 33(17), 2535-2552.

Palmer, S. (1999). Processing image structure. In: Vision science: photons to phenomenology. Cambridge: MA: MIT Press

Penn AA, R. P. (1998). Competition in retinogeniculate patterning driven by spontaneous activity. Science, 2108-12.

Penn AA, S. C. (1999). Brain waves and brain wiring: the role of endogenous and sensory-driven neural activity in development. Pediatr Res, 447-58.

Penn, A. (2001). Early brain wiring: activity-dependent processes. Schizophr Bull, $337-47$.

Penn, A. S. (s.f.)

Penn, A., \& Shatz, C. (2002). Principles of endogenous and sensory activitydependent brain development. New york: The newborn brain: neuroscience and clinical applications.

Peterka, R. (2002). Sensoriomotor integration in human postural control. J Neurophysiol, 88(3), 1099-1118.

Prieto, T., Myklebust, J., Hoffmann, R., Lovett, E., \& Myklebust, B. (1996). Measures of postural steadiness: differences between healthy young and elderly adults. IEEE Transactions on Biomedical Engineering, 43(9), 956-966.

Reeves, A. (2004). The visual neurosciences. Cambridge: MA: MIT Press.

Shannon, E., Skoczenski, A., \& Banks, M. (1996). Retinal illuminance and contrast sensitivity in human infants. Vision research, 36 (1), 67-76.

Shumway-Cook, A., \& Wollacott, M. (1995). Control de la postura y del equilibrio. En Control motor. Teorías y aplicaciones prácticas (págs. 101-121). Baltimore: William \& Wilkins

Sveistrup, H., \& Woollacott, M. (1996). Longitudinal development of the automatic postural response in infants. Journal of motor behavior, 28(1), 58-70.

Van Der Fits, I., \& Hadders-Algra, M. (1998). The development of postural response patterns during reaching in healthy infants. Neuroscience \& Biobehavioral, 22(4), 521-526

Wharrad, H., \& Davis, A. (1997). Behavioural and autonomic responses to sound in pre-term and full-term babies. British journal of audiology, 315-329.

White-Traut, R., Nelson, M., Burns, K., \& Cunningham, N. (1994). Environmental influences on the developing premature infant: Theoretical issues and applications to practice. Journal of Obstetric, Gynecologic, \& Neonatal Nursing, 23(5), 393-401.

Wilson, H. (1993 ). Theories of infant visual development. Early visual development: Normal and abnormal, 560-572.

Winter, D. (1995). Human balance and posture control during standing and walking. Gait \& Posture, 3(4), 193-214. 
Estabilidad postural en el neonato

Una revisión de la literatura 